\title{
The role of attractive forces in viscous liquids
}

\author{
Ludovic Berthier \\ Laboratoire Charles Coulomb, UMR 5221, CNRS and Université Montpellier 2, Montpellier, France \\ Gilles Tarjus \\ LPTMC, UMR 7600, CNRS and Université Pierre et Marie Curie, 4 Place Jussieu, 75252 Paris Cedex 05, France
}

(Dated: May 30, 2018)

\begin{abstract}
We present evidence from computer simulation that the slowdown of relaxation of a standard Lennard-Jones glass-forming liquid and that of its reduction to a model with truncated pair potentials without attractive tails is quantitatively and qualitatively different in the viscous regime. The pair structure of the two models is however very similar. This finding, which appears to contradict the common view that the physics of dense liquids is dominated by the steep repulsive forces between atoms, is characterized in detail, and its consequences are explored. Beyond the role of attractive forces themselves, a key aspect in explaining the differences in the dynamical behavior of the two models is the truncation of the interaction potentials beyond a cutoff at typical interatomic distance. This leads us to question the ability of the jamming scenario to describe the physics of glass-forming liquids and polymers.
\end{abstract}

PACS numbers: 05.10.-a, 05.20.Jj, 64.70.kj

\section{INTRODUCTION}

Since van der Waals, the statistical mechanics of liquids has been built on the idea that the structure of dense liquids is predominantly determined by the steep repulsive forces between molecules and that longer-ranged attractive interactions merely establish a cohesive background that affects the thermodynamics, but neither the structure nor the dynamics [1-4]. The physical rationale for this idea is that, in a dense liquid, density fluctuations coming from the displacement of the atoms or molecules involve the short-ranged forces. Longer-ranged forces, which are associated with a large number of particles, average to zero and have thus a negligible contribution to these fluctuations. This idea has been incorporated into an operational scheme by Weeks, Chandler and Andersen (WCA) [3] who proposed to split the intermolecular potential into a short-ranged repulsive component and a longer-ranged, more slowly varying component, and to treat the latter as a perturbation. This has provided a powerful tool to study liquids. Especially for simple atomic liquids, it has led to a very successful description of the structure, at the level of the static pair density correlations, and of the thermodynamics [3, 5].

As already understood in part by van der Waals, and stressed repeatedly in modern implementations [3, 5-7], this picture which neglects the fluctuations associated with the attractive, and more generally long-ranged, interactions becomes less accurate or even breaks down in some cases. For instance, when the liquid has large-size inhomogeneities, such as an interface, or when the attractive forces are short-ranged and directional, the net vector force on a given molecule or on a liquid domain that results from attraction with the rest of the system no longer vanishes. Then, a homogeneous, mean-field account of the attractive interactions is no longer valid. As is also well-known, on the low-density side of the liquid range, near the critical point or near the gas-liquid spinodal induced by attractive forces, density fluctuations develop on all lengthscales and become large, which again makes the mean-field description inappropriate.

If the van der Waals picture and the WCA theory of liquids have been extensively studied and tested as far as the pair structure and the thermodynamics are concerned, much less has been undertaken for the dynamics. Actually, the WCA theory and the associated division of the pair potential have not been submitted to systematic investigations $[8,9]$. We have recently revisited this point in the context of glass formation and found that a binary Lennard-Jones model and its WCA reduction to the repulsive components of the pair potentials, when studied at the same density, show very similar static pair density correlation functions but widely differing relaxation times as one lowers the temperature towards the glass phase [10]. In the present article, we expand on this first study and present a more exhaustive set of results for both the structure and the dynamics of the two liquid models. It confirms that a simple atomic glass-forming liquid in its viscous regime is manifestly an exception to the van der Waals picture for what concerns its dynamics, but not its pair structure. Although we cannot unambiguously assign this observation to a unique physical cause, it seems likely that it indicates that the viscous liquid is more heterogeneous than the mere consideration of its pair density correlation function would suggest.

In the previous short account of this work [10], we have described the effect of the attractive forces, included in the full Lennard-Jones model but not in its WCA reduction, as "nonperturbative" in the viscous liquid range. We address here more thoroughly this issue. We try to characterize the qualitative difference in dynamical behavior between the two models, by studying in particular the scaling of the relaxation time with density and temperature. This scaling appears as a ubiquitous (but 
approximate) property of glassforming liquids and polymers [11-14]; it is obeyed by the Lennard-Jones model but is strongly violated in the purely repulsive WCA model.

The presence or absence of density scaling in the dynamics of the binary Lennard-Jones liquid and its WCA reduction is clearly the consequence of the presence or absence of an attractive tail in the pair potentials of the two models. However, one should be cautious about the conclusion to be drawn from this fact. It is actually not obvious whether this is due to the attractive nature of the tail per se or to the introduction of a cutoff at a typical interatomic distance in the pair potentials, irrespective of the attractive or repulsive nature of the neglected longerranged interactions. Density scaling is indeed an exact property of purely repulsive power-law potentials, and it has been recently shown that both the structure and the dynamics of the binary Lennard-Jones model can be reproduced by a binary mixture of atoms with purely repulsive power-law interactions [15, 16]. On the other hand, as we shall also stress, density scaling is strongly violated, at low temperature and pressure, in systems of repulsive harmonic-like spheres, for which the pair potentials vanish beyond the typical interatomic distance [17, 18].

Building on these observations, we address the relevance of the "jamming scenario" 19] for describing the glass transition of liquids in their experimentally accessible range of density. This leads us to question the recently suggested equivalence between jamming phenomenon and colloidal glass transition on the one hand and glass transition of supercooled liquids on the other [20].

In Sec. II] we describe the technical detail of our work. In Sec. III we contrast the static and dynamic behavior of the two models under study. We study the scaling with density and temperature of the dynamics of the models in Sec. [V] and we address the role of attractive forces per se versus that of introducing a cutoff at a typical interatomic separation in the pair potential in Sec. V] Sec. VI is devoted to a discussion of the connection between glass-forming liquids and systems near jamming. Finally, we conclude the paper in Sec. VII.

\section{MODELS, SIMULATION, AND PHASE DIAGRAM}

\section{A. Models}

We compare the structure and the dynamics of a standard three-dimensional model of glass-forming liquid, the Kob-Andersen 80:20 binary Lennard-Jones mixture [21], and of its reduction to the purely repulsive part of the pair potentials proposed by WCA. In what follows, the former will be denoted by "LJ" and the latter by "WCA". The interatomic pair potential between species $\alpha$ and $\beta$,

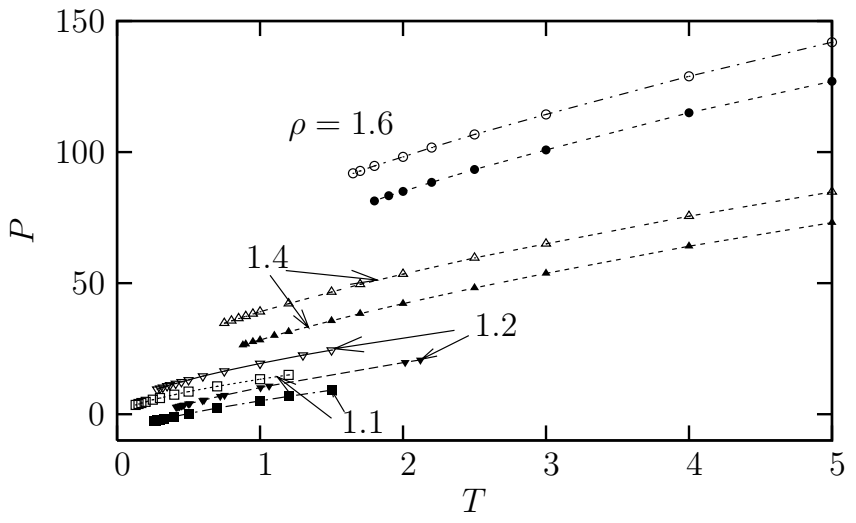

FIG. 1: The $P(T)$ equation of state for the LJ (filled symbols) and WCA (open sybols) models at different densities merely differ by a constant.

with $\alpha, \beta=A, B$, is given in the two systems by

$$
\begin{aligned}
v_{\alpha \beta}(r) & =4 \epsilon_{\alpha \beta}\left[\left(\frac{\sigma_{\alpha \beta}}{r}\right)^{12}-\left(\frac{\sigma_{\alpha \beta}}{r}\right)^{6}+C_{\alpha \beta}\right], \text { for } r \leq r_{\alpha \beta}^{c} \\
& =0, \text { for } r \geq r_{\alpha \beta}^{c},
\end{aligned}
$$

where $r_{\alpha \beta}^{c}$ is equal to the position of the minimum of $v_{\alpha \beta}(r)$ for the WCA model and to a conventional cutoff of $2.5 \sigma_{\alpha \beta}$ (merely introduced for practical reasons with no impact on the physical quantities) for the standard LJ model; $C_{\alpha \beta}$ is a constant that is fixed such that $v_{\alpha \beta}\left(r_{\alpha \beta}^{c}\right)=0$. The difference between the LJ and WCA potentials is a purely attractive contribution, dominated by the $r^{-6}$ contribution at large distance.

We have performed Molecular Dynamics simulations in the $N V E$ ensemble (after equilibration at a chosen temperature) with $N=900-1300$ particles (depending on density) and we have studied a broad range of density $\rho$ from 1.1 to 1.8. Lengths, temperatures and times are given in units of $\sigma_{A A}, \epsilon_{A A} / k_{B}$, and $\left(m \sigma_{A A}^{2} / 48 \epsilon_{A A}\right)^{1 / 2}$ respectively. It should be noted that the WCA truncated potential is continuous at the cutoff, but the resulting forces are not, which leads to a slow drift of the total energy during very long simulation runs in the microcanonical ensemble. To cure this problem without introducing random collisions or a thermostat, we periodically rescale velocities with a very low frequency to maintain the total energy constant [22].

To check the robustness of our results, we have also considered the two-dimensional version of the LJ and the WCA models, using a 65:35 mixture, because it is known to be less prone to crystallization in two dimensions than the 80:20 mixture [23].

\section{B. Thermodynamics and phase diagram}

In the WCA theory, the repulsive truncated model is taken as a reference for the full LJ model, from which one can determine the structure of the liquid, whereas 


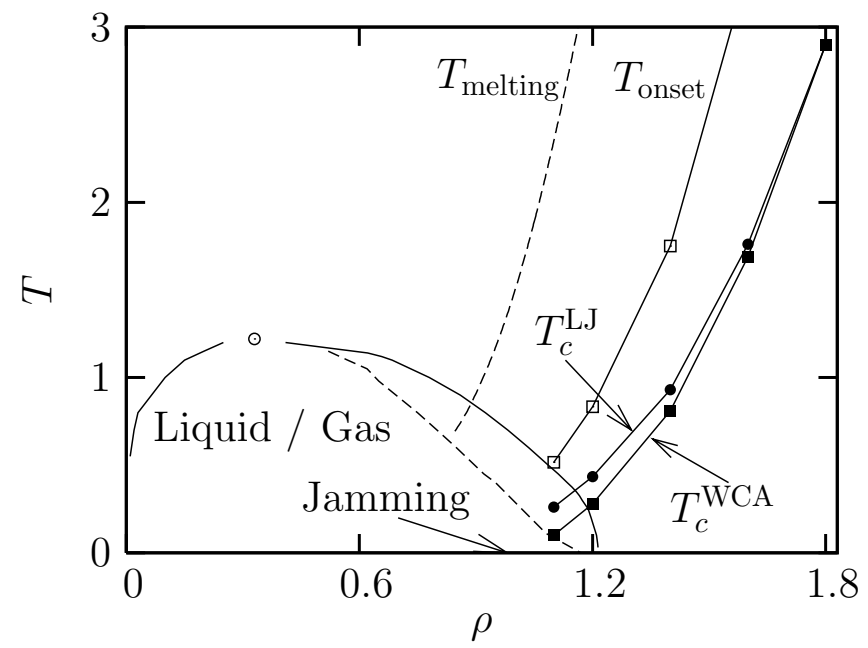

FIG. 2: Phase diagram of the system with: the melting line of the monodisperse LJ system [24], the coexistence curve 25], the spinodal line [26] and the onset temperatures $T_{\text {onset }}$ discussed in Sec. IV of the binary LJ mixture, the $T \rightarrow 0$ jamming limit of the WCA model; the mode-coupling transition line $T_{c}(\rho)$ as fitted from the numerical data [27].

the attractive component is treated as a perturbation that allows one to compute thermodynamic quantities. Within this description, the structure and the dynamics of the LJ and WCA models should be compared at the same $(\rho, T)$ state point. Their pressure then differs, with the attractive interaction roughly providing a negative background term. This is illustrated in Fig. 11 where we plot the simulation results for the equation of state, pressure versus temperature $P(T)$, of the two models at four densities from $\rho=1.1$ to $\rho=1.6$ : the pressure of the WCA model is roughly shifted up by a $\rho$-dependent constant from that of the full LJ model. As can be seen, the pressure of the full LJ mixture becomes negative at the lowest temperatures for $\rho=1.1$, which is the sign that the liquid is in a metastable state in the two-phase region inside the gas-liquid coexistence curve. The purely repulsive WCA model on the other hand has always a positive pressure and no coexistence region.

To help characterizing the different state points that will be considered, we give in Fig. 2 the phase diagram in the $(\rho, T)$ plane of the binary LJ model. For illustrating the relevant range for the dynamics, we also plot on this diagram the estimated $T_{c}$ of the mode-coupling theory as obtained from a fit to the simulation data [27]. For the purpose of this work, the merit of such a fit is immaterial, the $T_{c}$ line being a convenient indicator of a temperature scale where dynamics has slowed down by about 4 decades as compared to the high-temperature fluid. The mode-coupling line ends up for $\rho=1.1$ in the metastable liquid, as mentioned above, whereas densities $\rho=1.6$ and $\rho=1.8$ are clearly outside the conventional liquid range (one is always much above the critical temperature). These densities actually correspond to conditions that, although easily investigated in simula-

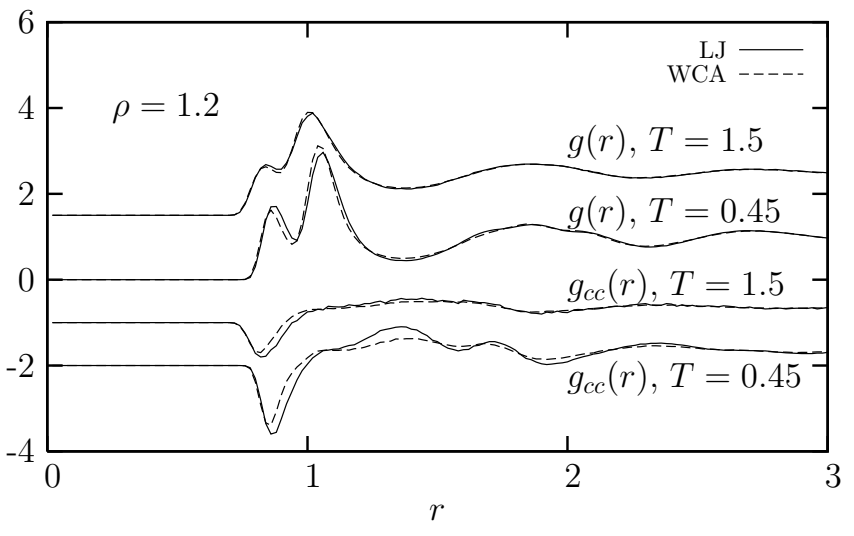

FIG. 3: Pair correlation functions of the (total) density fluctuations $g(r)$ and of the concentration fluctuations $g_{c c}(r)$ for the LJ and WCA liquids at high and low temperature for the canonical liquid density $\rho=1.2$. The different curves are vertically shifted, for clarity.

tions of simple models, are not experimentally relevant, even in high-pressure experiments on glass-forming liquids (see also Ref. [28]). The density $\rho=1.2$ is the standard condition at which the slowdown of relaxation of the binary Lennard-Jones mixture is usually considered [21]. It corresponds to typical liquid (and viscous liquid) states. Most of our simulations are performed in the viscous regime in the region delimited by the onset temperature $T_{\text {onset }}$ and the mode-coupling lines in Fig. 2 ,

As for the WCA model, because of the absence of attractive interactions, it does not have a gas-liquid transition and could in principle be studied at all densities down to zero temperature. In practice however, we found that the system crystallizes in simulations when cooled down to small but nonzero $T$ at densities below $\rho \simeq 1$. and this is the reason which prevents us from extending the mode-coupling line down to $T=0$. On the other hand, directly at $T=0$, the WCA model could in principle be subjected to various "jamming" protocols with density as the control variable [29]. We shall come back to these aspects in the following.

\section{CONTRASTING THE BEHAVIOR OF THE PAIR STRUCTURE AND OF THE DYNAMICS}

\section{A. Pair correlation functions}

We first consider the structure of the two liquid models, as characterized by the static two-body density correlations. We focus here on the pair correlation functions $g_{\alpha \beta}(r)$, the static structure factors $S_{\alpha \beta}(q)$ having already been displayed elsewhere [27].

In Ref. [10], we have shown that the pair correlation function of the (total) density fluctuations,

$$
g(r) \equiv x_{A}^{2} g_{A A}(r)+2 x_{A} x_{B} g_{A B}(r)+x_{B}^{2} g_{B B}(r),
$$



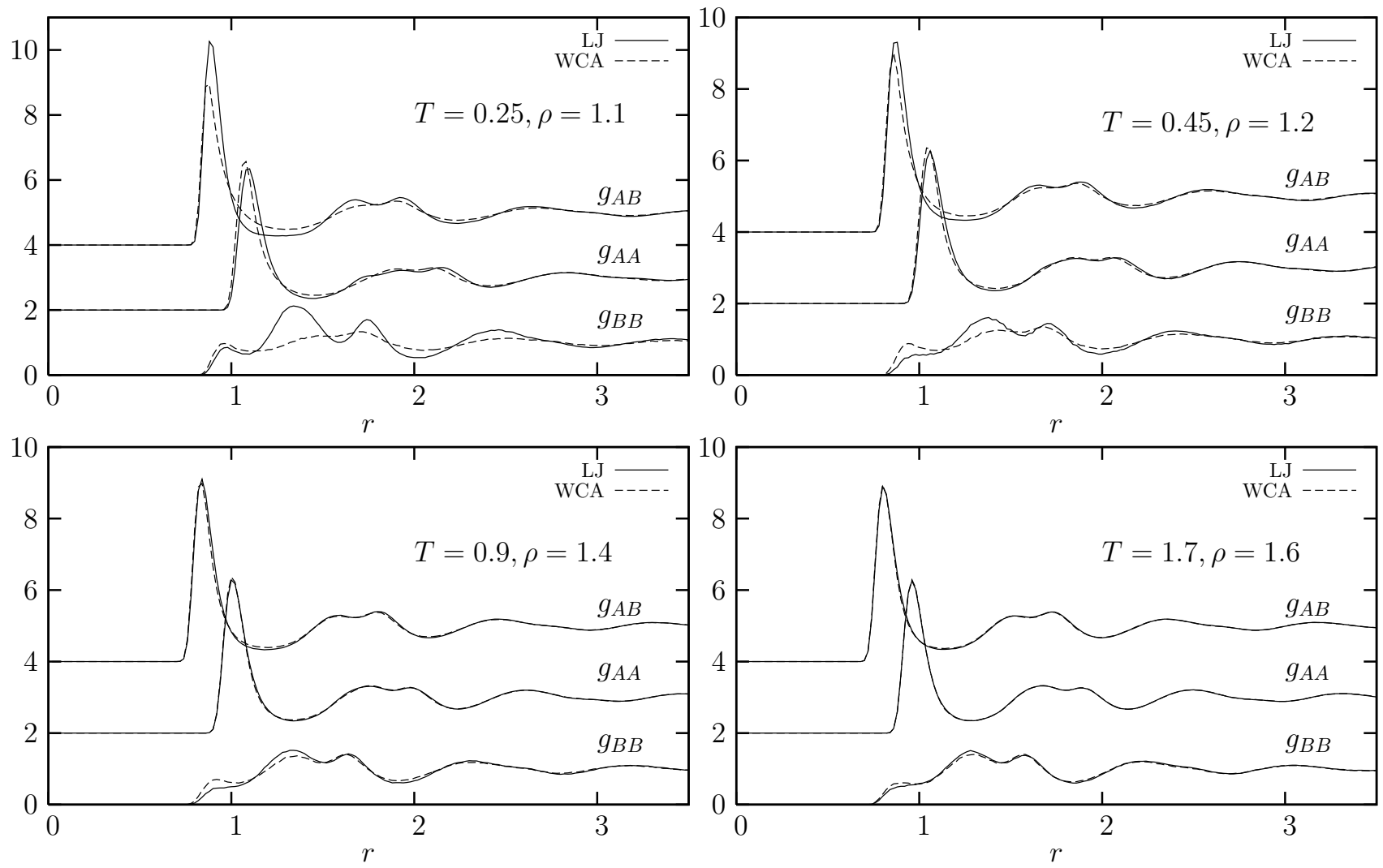

FIG. 4: Evolution with density of the partial pair correlation functions of the LJ and WCA liquids at low temperature and various densities. The different curves are vertically shifted, for clarity.

where $x_{\alpha}$ denotes the concentration of species $\alpha$, is extremely similar for the LJ and the WCA models and that its shape varies only weakly with temperature. This is illustrated in Fig. 3 for the canonical density $\rho=1.2$. Weeks, Chandler and Andersen [3] pointed out that, in the case of a binary mixture, the coincidence between the structure of the two models should not be as good for the pair correlations involving the fluctuations of concentration $\left(\delta c=x_{B} \delta \rho_{A}-x_{A} \delta \rho_{B}\right)$, as those are less constrained at high density than the (total) density fluctuations $\left(\delta \rho=x_{A} \delta \rho_{A}+x_{B} \delta \rho_{B}\right)$. We have checked this by plotting the pair correlation function of the concentration fluctuations, $g_{c c}(r)$, defined as

$$
g_{c c}(r) \equiv x_{B}^{2} g_{A A}(r)-2 x_{A} x_{B} g_{A B}(r)+x_{A}^{2} g_{B B}(r) .
$$

The results are also shown in Fig. 3. As anticipated, the difference between the LJ and WCA models is still weak but more pronounced for $g_{c c}(r)$ than for $g(r)$.

Finally, we display in Fig. 4 the evolution with density of the partial pair correlation functions $g_{\alpha \beta}(r)$ for the two liquid models. As there is less difference between the two models at high temperature, we concentrate on the low temperatures, thus emphasizing those state points where differences are more pronounced. At high density, all curves nearly superimpose, but one can see notable differences between the two models at $\rho=1.1$, especially for the correlation functions involving the minority species $B$. At such a density, attractive interactions start playing a nonnegligible role as the full LJ system enters the metastable liquid region inside the coexistence curve and approaches the spinodal where density fluctuations grow very large.

It is perharps not surprising that $B B$ correlations are more affected by removing the attractive part of the potential, as the interaction parameters of the model were specifically chosen to favor attraction between $A B$ particles, such that $B$ particles can efficiently frustrate the crystallization of the majority $A$ component. By removing the attractive interactions, we see that the first peak of $g_{B B}(r)$ is more pronounced for the WCA model, while the first peak in $g_{A B}(r)$ is less pronounced, showing that the effect engineered by Kob and Andersen is less efficient. This also explains why the present WCA mixture crystallizes more easily at low density than its LJ counterpart. Note finally that $g_{B B}(r)$ dominates the behavior of $g_{c c}(r)$, see Eq. (3), which explains why $g_{c c}(r)$ is a more sensitive probe of the structural differences of the two models than $g(r)$, which is dominated by $g_{A A}(r)$.

Although we discussed in detail these small differences, our results on the whole provide one more confirmation of the validity of the WCA theory for the equilibrium pair structure of dense liquids. The rather small differences observed between the LJ and WCA models could probably be captured by a perturbative treatment of the 

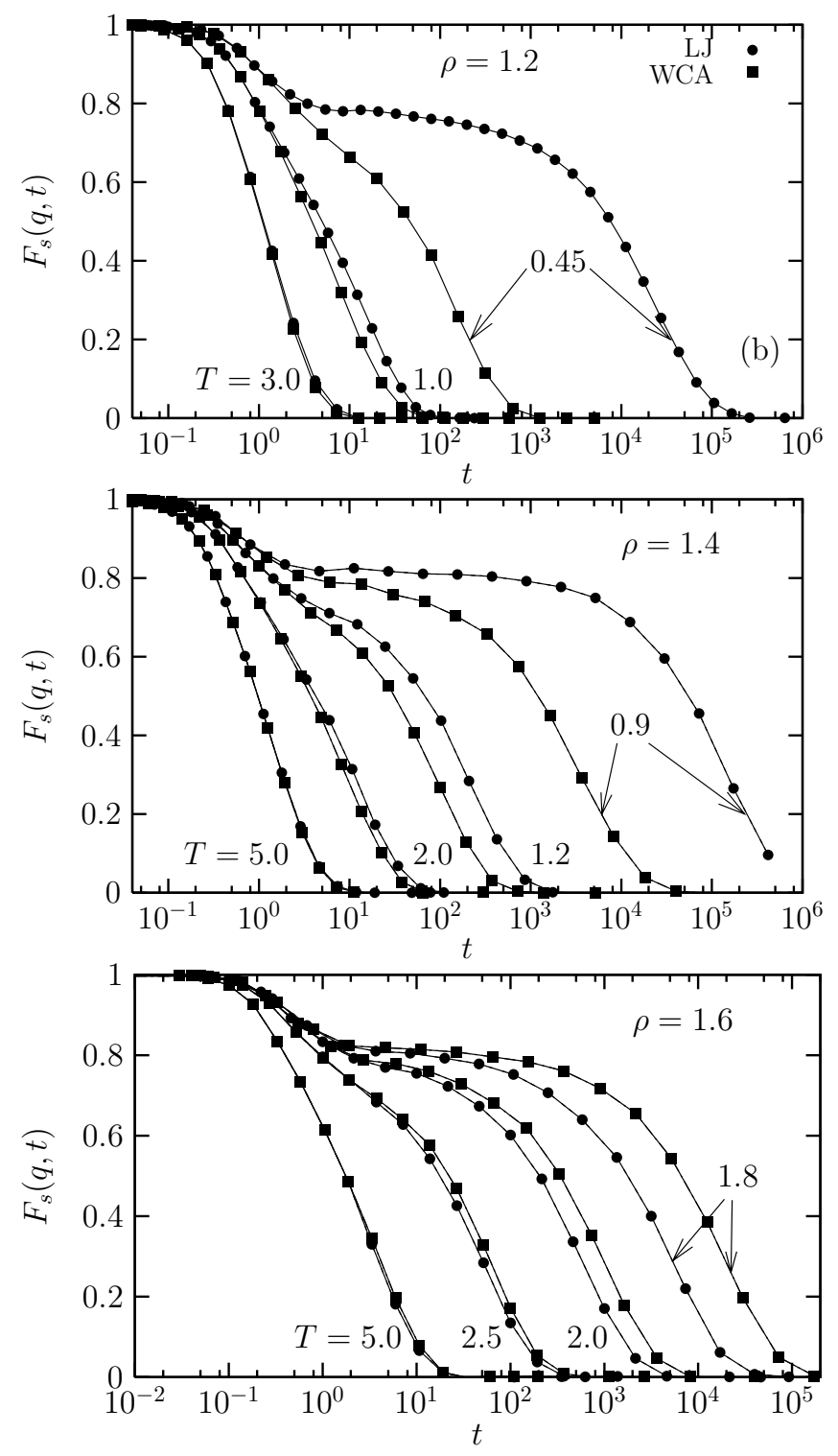

FIG. 5: Comparison of the time dependence of the selfintermediate scattering function $F_{s}(q, t)$ for $q \sigma_{A A} \simeq 7.2$ of the LJ and WCA models at the same temperatures, shown for different densities.

attractive tail, for instance along the lines suggested in Ref. 30].

\section{B. Dynamic correlations and timescales}

We now turn to the results concerning the dynamics of the two liquid models. As already shown in our previous publication [10], the idea that the dynamical behavior of a liquid is essentially insensitive to longer-ranged attractive interactions breaks down in an increasingly spectacular manner as temperature decreases. The difference between the liquid in the presence and in the absence of the attractive tail is small but already noticeable at high
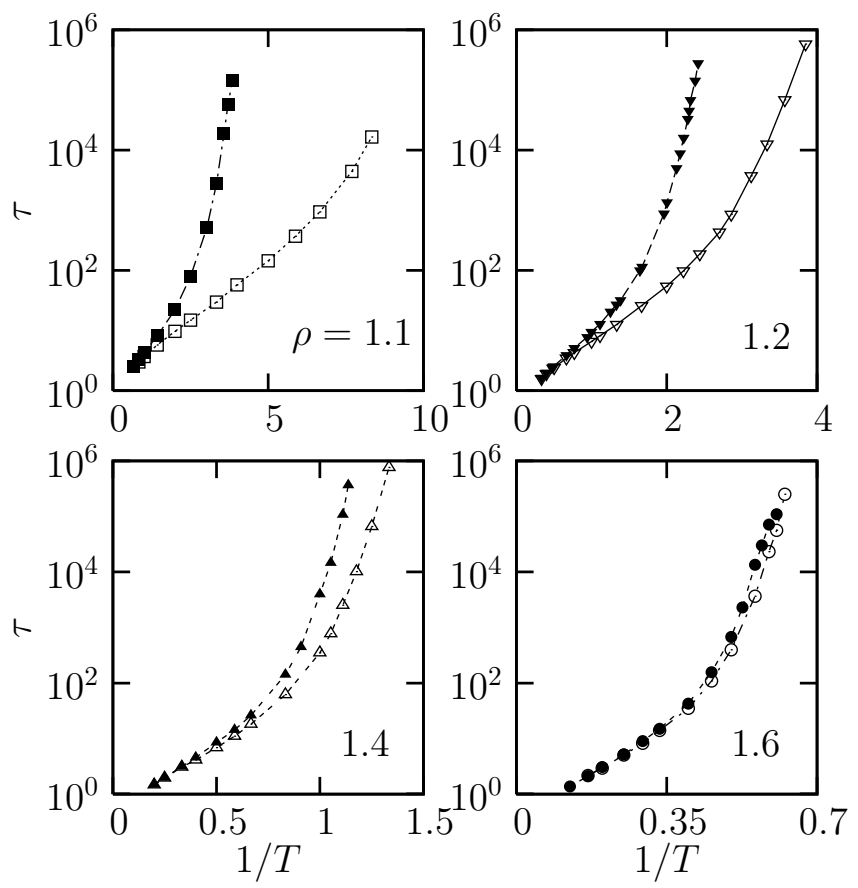

FIG. 6: Arrhenius plot of the relaxation time of the LJ (filled symbols) and WCA (open symbols) models for several densities. Note that the temperature range is different for each panel.

temperature in the "normal" range (see also Refs. 8, 9]) and it rapidly builds up in the low-temperature regime relevant for the glass transition phenomenon [10]. Here, we analyze in more detail the influence of the density.

To analyze the dynamics of the models, we have mostly studied the time dependence of the self-intermediate scattering functions defined by

$$
F_{s}(q, t)=\frac{1}{N}\left\langle\sum_{j=1}^{N} e^{i \mathbf{q} \cdot\left(\mathbf{r}_{j}(t)-\mathbf{r}_{j}(0)\right)}\right\rangle,
$$

with $q \sigma_{A A} \simeq 7.2$, which we kept constant for all state points. This corresponds roughly to studying single particle displacements over a length scale comparable to the inter-particle distance. Representative data points are shown in Fig. 5 for three densities and a broad range of temperatures. These figures strikingly illustrates how the small differences in the relaxation dynamics of the high-temperature liquids become a dramatic effect at low temperatures. For $\rho=1.2$ and $T=0.45$ for instance, the LJ system hardly relaxes in the timescale of the simulation while this temperature corresponds to a modestly supercooled state. When $\rho$ increases, these differences decrease, but note that even for $\rho=1.6$ and $T=1.8$ (a temperature which is $50 \%$ larger than the critical point, see Fig. 2) the time correlation functions still differ by a large factor.

From the time decay of the self-intermediate scattering function, we extract a relaxation time, $\tau$, defined in practice as $F_{s}(q, \tau)=\exp (-1)$. We show in Fig. [6] an Ar- 

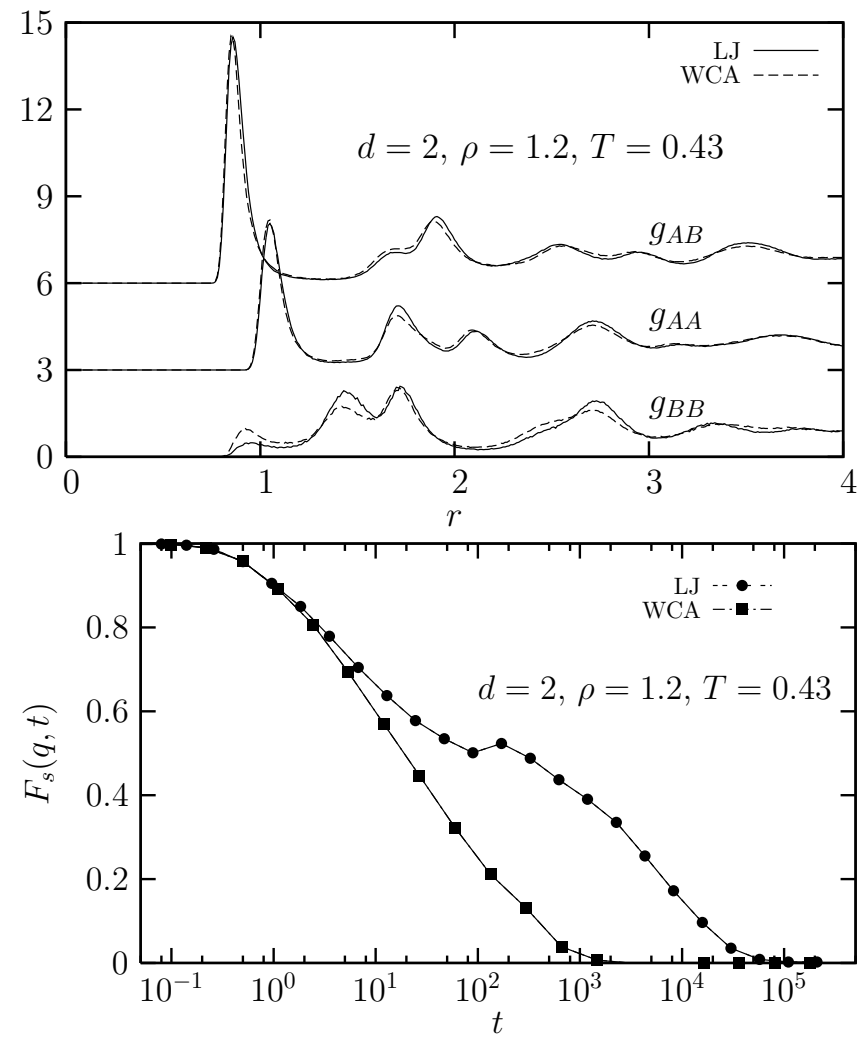

FIG. 7: Two-dimensional 65:35 Kob-Andersen binary LJ mixture and its WCA reduction: Top: Partial pair correlation functions $g_{\alpha \beta}(r)$ for a typical liquid density $\rho=1.2$ at low temperature. Bottom: Time dependence of the self intermediate scattering functions under the same conditions.

rhenius plot of this relaxation time for several densities. The figure clearly illustrates that the dramatic difference between the two models that is seen at liquid densities (with already a difference of 3 orders of magnitude at the lowest temperature at which we can equilibrate the full binary LJ model) decreases slowly with density. In such a logarithmic representation covering many decades, the presence or absence of the attractive tail of the potentials appears irrelevant at very high densities only. However, as stressed above, such densities are not realistic for actual glass-forming liquids.

\section{Two-dimensional case and other examples}

We note in passing that the pattern that we have found here, namely that the WCA reduction of the binary LJ mixture to a truncated purely repulsive systems has virtually no effect on the pair correlations of the total density but strongly affects the dynamics, is not unique. We have also obtained the same effect for instance in a two-dimensional version of the binary 65:35 LJ mixture model, as illustrated in Fig. 7 .

We point out that, although not appreciated before, the conclusion that the WCA truncation of Lennard-
Jones forces has a dramatic impact on the dynamics can in fact be drawn by comparing the results already published in the literature for different Lennard- Jones mixtures [21, 31] and for their WCA truncations [32, 33].

Additionnally, since their initial publication [10], our results for the three-dimensional Kob-Andersen 80:20 mixture have now been confirmed by independent studies [15, 16].

\section{To be, or not to be (perturbative)}

Before closing this section, we would like to discuss how to best characterize the difference in the dynamical behavior of the LJ and WCA models at liquid densities and what to make of it. In our earlier publication [10], we have described the effect of the attractive tail of the pair potentials on the dynamics as "nonperturbative". At a quantitative level, the gap between the characteristic time scales of the two models indeed becomes enormous and widens as temperature decreases. By itself, this observation undoubtedly invalids any claim that attractive tails can be neglected when describing the dynamics (the relevance, or not, of the attractive nature of the tail will be discussed in section (V), but it is not enough to justify the qualifier "nonperturbative". In principle, this quantitative difference, no matter how large, could be scaled out through the introduction of parameters which themselves could be computed via a perturbative treatment of the longer-ranged interactions. This would be true for instance if the dynamics could be predicted on the sole basis of the knowledge of the pair structure, with minor effects in the latter being strongly amplified in the former. However, we show elsewhere [34] that such approaches are unable to account for the diverging behavior of the LJ and WCA liquids in the viscous regime as temperature is lowered. In addition, we have found evidence that the difference in the dynamics of the two systems is not only quantitative but also qualitative. This is the point we now discuss in more detail.

\section{ON THE DENSITY SCALING FOR THE RELAXATION TIME}

\section{A. Density scaling in supercooled liquids and polymers}

The simplest way for trying to scale out the difference in the $T$-dependences of the LJ and WCA liquids is to renormalize the temperature by one density-dependent parameter chosen to make the curves of the two models coincide at high temperature, where a perturbative treatment of the attractive tails more likely to apply.

The most convenient way to proceed is to fit the high- $T$ data to an Arrhenius form,

$$
\tau(\rho, T) \approx \tau_{\infty}(\rho) \exp \left[E_{\infty}(\rho) / T\right]
$$


which indeed provides a good empirical description, irrespective of the physical meaning one is willing to put in its use. This procedure suggests to rescale $T$ for the whole range under study by the extracted energy parameter $E_{\infty}(\rho)$. When applied to glass-forming liquids and polymers [11], this procedure has been shown to provide a very good collapse of the relaxation and viscosity data at different densities, according to

$$
\frac{\tau(\rho, T)}{\tau_{\infty}}=\mathcal{F}_{1}\left[\frac{E_{\infty}(\rho)}{T}\right] .
$$

With specific functional forms for the density-dependent scaling parameter (see also below), this relation has been successfully applied to an impressive variety of glassforming liquids and polymers [11 14]. Thus, there is enough empirical evidence to take this scaling property as a genuine characteristic of glass formation in these systems.

It must be noted that Eq. (6) is far from being trivial, as it connects the dynamics in the high-temperature liquids to the behaviour found in the viscous, low temperature regime. More precisely, Eq. (6) shows that two physical quantities are in fact proportional: the hightemperature activation energy, $E_{\infty}$, and the "onset temperature" for slow dynamics, $T_{\text {onset }}$, which could be operationally defined at the point where $\mathcal{F}_{1}(x)$ departs from its high- $T$ (Arrhenius) behavior:

$$
T_{\text {onset }}(\rho) \propto E_{\infty}(\rho) .
$$

Physically, $T_{\text {onset }}$ marks the temperature below which the liquid starts behaving in a more collective and heterogeneous manner 35 38].

As expected, and already displayed in Ref. [10], the data for the full LJ binary mixture obey very well the density scaling of Eq. (6), see Fig. 2a of Ref. [10] (a small deviation from the data collapse can be observed at the lowest density $\rho=1.1$ corresponding to the metastable liquid inside the gas-liquid coexistence curve). On the other hand, the scaling relation is strongly violated for the WCA liquid: see Fig. 2b of Ref. [10].

One important consequence is that the isochoric fragility, which characterizes the steepness of the $T$ driven slowdown of relaxation and can be quantified by the derivative $\partial \log \left[\tau(\rho, T) / \tau_{\infty}\right] / \partial \log T$ taken at constant $\rho$ and evaluated at the glass transition (or for any given value of $\tau / \tau_{\infty}$ ), is essentially independent of density for the LJ model, as also found in real glass-forming liquids and polymers [1]], but strongly depends on density for the WCA model. This presence or absence of (even approximate) density scaling represents, we claim, a qualitative difference between the two liquid models.

\section{B. Empirical data collapse}

If one wishes to also collapse the relaxation data at all densities for the WCA model, one must introduce at
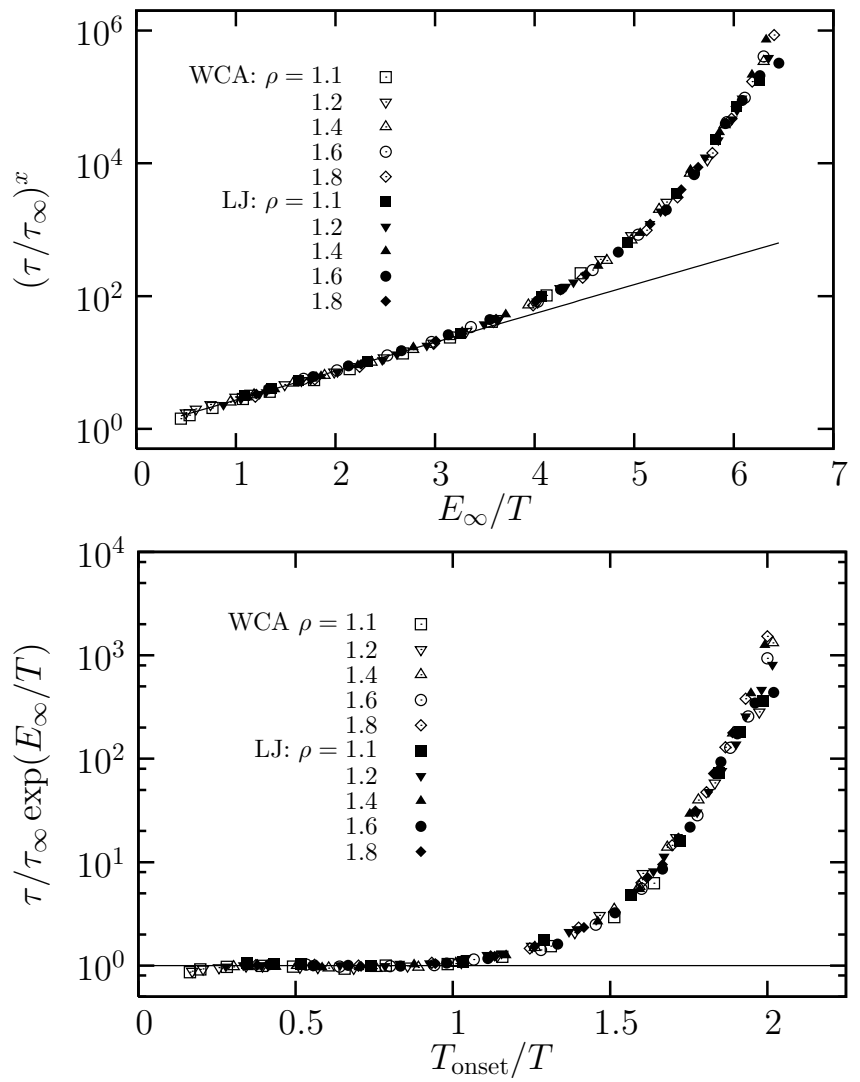

FIG. 8: Empirical collapse of the relaxation-time data for the LJ and WCA models at densities from $\rho=1.1$ to $\rho=1.8$. Top: Using Eq. (9) with parameters listed in Table 1 Bottom: Using Eq. (8) with parameters listed in Table प. The absolute scale of $y(\rho)$ is chosen so that the deviation from the high- $T$ behavior takes place around $T_{\text {onset }}=y E_{\infty} \approx 1$.

least one additional, $\rho$-dependent parameter. This procedure may have only little physical significance per se, but it allows one to quantify and discuss more precisely the deviation from density scaling, which is obeyed by the LJ model only.

We have found that the two functional forms,

$$
\frac{\tau(\rho, T)}{\tau_{\infty} \exp \left(E_{\infty}(\rho) / T\right)}=\mathcal{G}_{1}\left[y(\rho) \frac{E_{\infty}(\rho)}{T}\right]
$$

and

$$
\left(\frac{\tau(\rho, T)}{\tau_{\infty}}\right)^{x(\rho)}=\mathcal{G}_{2}\left[\frac{E_{\infty}(\rho)}{T}\right],
$$

both lead to an excellent collapse on a master-curve of the LJ and WCA data, as seen in Fig. 8, It is straightforward to show that the density scaling of Eq. (6) is recovered when either $x(\rho)$ or $y(\rho)$ is independent of density.

We arrived at the formula in Eqs. (8) 9) by using the possibility that Eq. (77) is not valid and that one needs two distinct energy scales to describe the behaviour of the WCA model. This additional freedom can be incorporated in a variety of ways, and we present two of them 


\begin{tabular}{||l||l|l|l|l||l|l|l|l||} 
& WCA & & & & LJ & & & \\
\hline$\rho$ & $T_{\text {onset }}$ & $E_{\infty}$ & $y$ & $x$ & $T_{\text {onset }}$ & $E_{\infty}$ & $y$ & $x$ \\
\hline 1.1 & 0.197 & 0.885 & 4.49 & 0.586 & 0.517 & 1.6 & 3.09 & 1 \\
1.2 & 0.513 & 2. & 3.89 & 0.685 & 0.833 & 2.55 & 3.06 & 1 \\
1.4 & 1.51 & 5.25 & 3.47 & 0.897 & 1.75 & 5.6 & 3.20 & 1 \\
1.6 & 3.2 & 10.6 & 3.31 & 0.958 & 3.33 & 10.9 & 3.27 & 1 \\
1.8 & 5.6 & 18. & 3.21 & 1.0 & 5.67 & 18 & 3.17 & 1 \\
\hline
\end{tabular}

TABLE I: The parameters for the empirical data collapse. The parameter $y$ is equal to the ratio between $E_{\infty}$ and $T_{\text {onset }}$. It fluctuates around $\approx 3.2$ for LJ at all densities, and decreases strongly with $\rho$ for the WCA.

in Fig. 8, In a recent work [39], our published data [10] for the LJ and WCA models have been re-analyzed and collapsed for all densities by means of a specific functional form which similarly makes use of two independent energy scales; however, this collapse only holds in the viscous regime, while we also consider the high-temperature regime in Fig. 8

As expected, we find that for the LJ model, the two additional parameters $y(\rho)$ and $x(\rho)$ are indeed essentially independent of density. While $x=1$ is used in Fig. 8 , the $y$ values are given in Table I. with small variations from a constant that have no systematic dependence on density within statistical accuracy. On the contrary, to collapse also the data for the WCA model, significant deviations from constant behavior (with systematic trends as a function of density) are needed for both $x(\rho)$ and $y(\rho)$, see Table【.

The presence or absence of density scaling for LJ and WCA models is a qualitative effect, which we have empirically quantified by introducing the parameter $x$ or $y$ in Eqs. [8, 91). As pointed out in Ref. [39], the differences found in the parameters obtained for the two models are modest ( $x$ or $y$ vary by about $50 \%$ ) compared to the observed differences in the relaxation times themselves; but this is of course expected for parameters characterizing the temperature dependence of the logarithm of the relaxation times, and this does not contradict our conclusion that the presence or absence of density scaling represents a qualitative feature of the slowing down of a glass-former.

From Eq. (8) we see that a sensible definition of the onset temperature becomes

$$
T_{\text {onset }}(\rho) \propto y(\rho) E_{\infty}(\rho) .
$$

This is nicely illustrated in Fig. 8 (bottom) where $T_{\text {onset }}$ can be estimated as the point at which the relaxation data starts to significantly deviate from the high- $T$ behavior; the resulting values of $T_{\text {onset }}$ are given in Table I The physical significance of the values obtained in this way are independently confirmed by checking that $T_{\text {onset }}$ also corresponds to the crossover at which the $T$-dependences of the self-diffusion constant, $D_{\text {self }}(\rho, T)$, and of the relaxation data, $\tau(\rho, T)$, begin to split, see Fig. 9 for an illustration at $\rho=1.2$. These values are independently confirmed in Ref. [40].

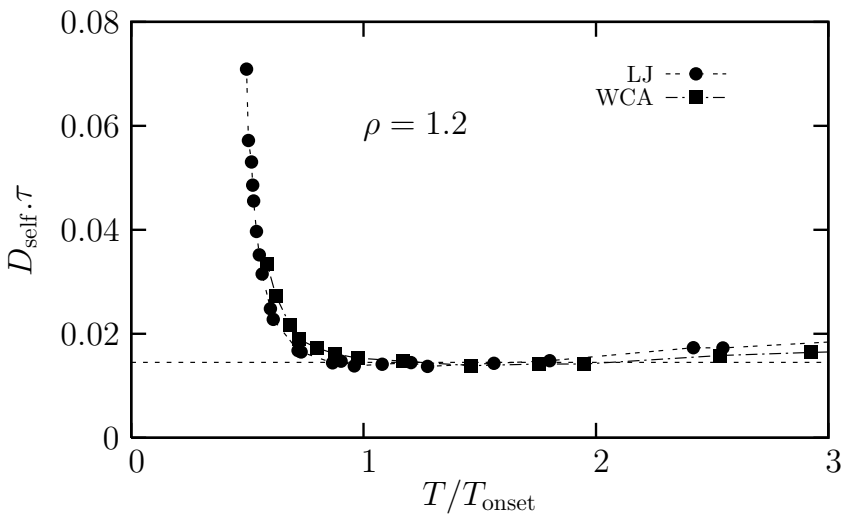

FIG. 9: Decoupling between the $T$ dependences of the self diffusion constant $D_{\text {self }}$ and of the relaxation time $\tau$ for $\rho=1.2$ as a function of the temperature $T$ rescaled by the independently measured $T_{\text {onset }}$. For both models, the product $D_{\text {self }} \tau$ begins to increase below $T \approx T_{\text {onset }}$, which differ by a factor 1.6 for both models, see Table $\llbracket$

The data compiled in Table \actually imply that the LJ and WCA models have distinct dynamics in the hightemperature regime already since $E_{\infty}$ of both models only become equal at very high density. Both models are also characterized by distinct onset temperatures, which differ by a factor $\approx 1.6$ at $\rho=1.2$, and, again, slowly converge to similar values at large density. Finally, the qualitative character of the impact of the truncation of the potential on the slow dynamics is illustrated by the fact that the isochoric fragility of the LJ model is independent of density while fragility strongly increases with density for the WCA model, as parametrized by the evolution of either $x(\rho)$ or $y(\rho)$ which is qualitatively different for both models.

\section{Analogy with power-law repulsive spheres}

In the case of the LJ liquid, the density scaling can be expressed, with essentially as good data collapse as with Eq. (6), by using a power-law density dependence for the scaling parameter (see Fig. 2c of Ref. [10] showing that $\left.E_{\infty}(\rho) \sim \rho^{5}\right)$, namely,

$$
\frac{\tau(\rho, T)}{\tau_{\infty}}=\mathcal{F}_{2}\left[\frac{\rho^{\gamma}}{T}\right]
$$

with $\gamma \simeq 5$. In this form, density scaling was argued to be related to the property that LJ models are "strongly correlating liquids" [41], which means that the fluctuations in the potential energy,

$$
U=\sum_{i<j} v\left(\left|\mathbf{r}_{i}-\mathbf{r}_{j}\right|\right)
$$

and those in the virial part of the pressure,

$$
W=-\frac{1}{3} \sum_{i<j} w\left(\left|\mathbf{r}_{i}-\mathbf{r}_{j}\right|\right),
$$


with $w(r)=r v^{\prime}(r)$, are correlated at all state points. The correlation is characterized by a single parameter, $\Gamma$, defined by a linear fit through a scatter plot of the time fluctuations of $U$ and $W$. The connection between the density scaling of the dynamics and the strong-correlation property of the fluctuations shows up in the approximate relation: $\gamma \simeq \Gamma$.

The rationale which has first been put forward to justify both properties [14, 15, 41] is as follows. A dense LJ liquid is dominated by the repulsive forces (the van der Waals picture of liquids), but the relevant part of the repulsive interaction involves interatomic distances in the range near to (but less than) the minimum of the pair potential; in this range, the potential looks like an effective power law $r^{-3 \gamma}$ with an effective repulsion which is steeper than the bare $r^{-12}$ repulsive component of the LJ potential, i.e. $3 \gamma>12$. In this picture, the LJ model essentially behaves as a repulsive $r^{-3 \gamma}$ power-law interacting model, a model that is known to rigorously lead to the two considered properties, with indeed a strict equality $\gamma=\Gamma$.

The simple rationale given above has however a major flaw: it predicts that the WCA and the full LJ models should behave in exactly the same way as their pair potentials coincide for distances shorter than the minimum. This, as shown above, is clearly wrong. Thus, the WCA-like argument that the fluctuations in dense Lennard-Jones liquids (density scaling and $U-W$ correlation) can be understood on the basis of the steep repulsive core only is, in general, not valid.

An improvement over the argument was later suggested by Dyre and coworkers [42]. They argued that the relevant interatomic distances actually include the vicinity of the minimum on both sides of it and that a better description of the LJ potential is through a power law complemented by a linear term; as the latter has essentially no effect on the fluctuations at constant density, the fluctuation properties of the full LJ system are thus predicted to be those of a properly adjusted power-law repulsive potential. The WCA truncated potential being indeed less well described by a power law plus a linear term (for distances above the minimum), this argument could allow one to sidestep the criticism raised above.

We have repeated the analysis of the correlation between the fluctuations of the potential energy and those of the virial for the two models. We find that, as expected from the work of Dyre and coworkers, the LJ model has a strong correlation at any given density. The WCA model also has strong correlations between energy and virial at any given $(\rho, T)$ state point [43], as illustrated in Fig. 10 for $\rho=1.2$ and $T=0.45$.

The parameter $\Gamma$ that we extract from the slope of the correlation plot is reported in Fig. 10 for a broad range of state points. It is virtually independent of temperature and weakly dependent on density in the LJ case, but has a stronger density dependence (and temperature dependence when $\rho=1.2$ ) in the WCA case (see also Ref. [15]). Therefore, if one neglects the modest density
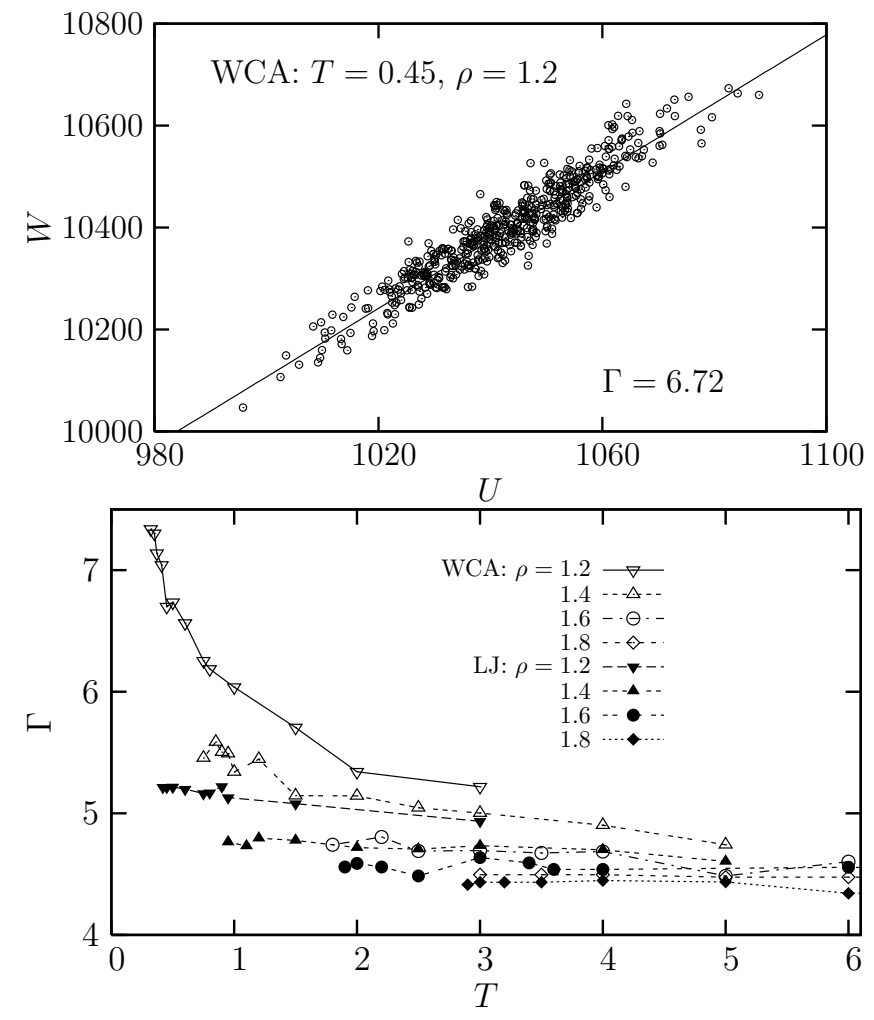

FIG. 10: Top: Linear correlation between the fluctuations of the virial $W$ and the fluctuations of the potential energy $U$ for the WCA liquid at $\rho=1.2$ and $T=0.45$, with a slope $\Gamma$. Bottom: Variation with temperature of the slope $\Gamma$ of the correlation between the fluctuations of $W$ and $U$ for the LJ and the WCA liquids at several densities between 1.2 and 1.8.

dependence of $\Gamma$ for the LJ model (in Fig. 10, the effective slope $\Gamma$ varies between 4.5 and 5.2 over a wide range of density and temperature), one can indeed conclude that the latter is "strongly correlating" over the whole liquid range with an effective, constant, value of $\Gamma$ of about 5 . Of course, the data in Fig. 10 underlie that there is still some arbitrariness in the choice of the value of $\Gamma$, and the quality of the density scaling depends on this choice.

On the other hand, as shown in Fig.10, the variation of $\Gamma$ in the WCA liquid is probably too large to be replaced by a single, effective constant. In this case, neither the fluctuations nor the dynamics are purely determined by reducing the pair potentials to repulsive power-law ones with an exponent chosen to reproduce the effective steepness of the potential near the minimum. Thus, although both phenomena (density scaling and strong-correlation property) seem connected to each other, the example of the two models presented in this study shows that it is not obvious to know a priori for which materials these observations may apply or not. 


\section{IS THE ABSENCE OR PRESENCE OF (APPROXIMATE) SCALING DUE TO ATTRACTION OR TO TRUNCATION IN THE POTENTIAL?}

We have seen that the presence (in the LJ model) or the absence (in the WCA model) of an attractive tail in the pair potentials has a large quantitative influence on the dynamics and strong consequences for the fluctuations and the relaxation properties of the liquids. In particular the (approximate) density scaling observed for the temperature dependence of the relaxation time in glassforming liquids and polymers (with the resulting density independence of the isochoric fragility) is found in the LJ model but not in the WCA one. A central question then to be raised is whether the differences stem from the attractive character of the tail per se or from the truncation of the range of the potential to typical interatomic distances.

A first hint that truncation is the key feature is provided by looking at the behavior of systems of spheres with purely repulsive power-law potentials. As already mentioned, such liquids are (rigorously) "strongly correlating" and show an exact density scaling of the relaxation time, as a consequence of the scale-free power law behavior of the potential. This example evidences that the presence of attractive interactions is not a necessary ingredient in establishing these properties.

Additional evidence along the same lines has recently been provided by Pedersen et al. [16] who showed that the pair structure and the dynamics of the binary LJ mixture at liquid densities can be very well reproduced by replacing the LJ pair potentials by nontruncated powerlaw repulsive potentials with an appropriately adjusted exponent. Note that they chose a repulsion of the form $r^{-15.48}$ such that $\gamma=\Gamma=5.16$, in rough agreement with the data shown in previous sections.

To confirm that truncating the potentials beyond a cutoff of the order of typical interatomic distances is responsible for the absence of density scaling seen in the WCA model, we revisit the example of fluid mixtures of repulsive harmonic spheres, with pair potentials

$$
\begin{aligned}
v_{\alpha \beta}(r) & =\frac{\epsilon_{\alpha \beta}}{s}\left(1-\frac{r}{\sigma_{\alpha \beta}}\right)^{s}, \text { for } r \leq \sigma_{\alpha \beta} \\
& =0, \text { for } r \geq \sigma_{\alpha \beta},
\end{aligned}
$$

where $s=2$; these systems are commonly used in the context of zero-temperature jamming phenomena [29]. A wide range of temperature and density was studied by computer simulation in Refs. [17, 18. . As noticed in these references, the isochoric fragility of the system strongly depends on density. We have replotted in Fig. 11 the relaxation-time data in the way described in section IV (and used in Fig. 2 of Ref. [10]) by rescaling the temperature with a density-dependent energy parameter $E_{\infty}(\rho)$ chosen to make the high- $T$ data collapse on a single curve. This plot clearly shows that, just like the WCA model

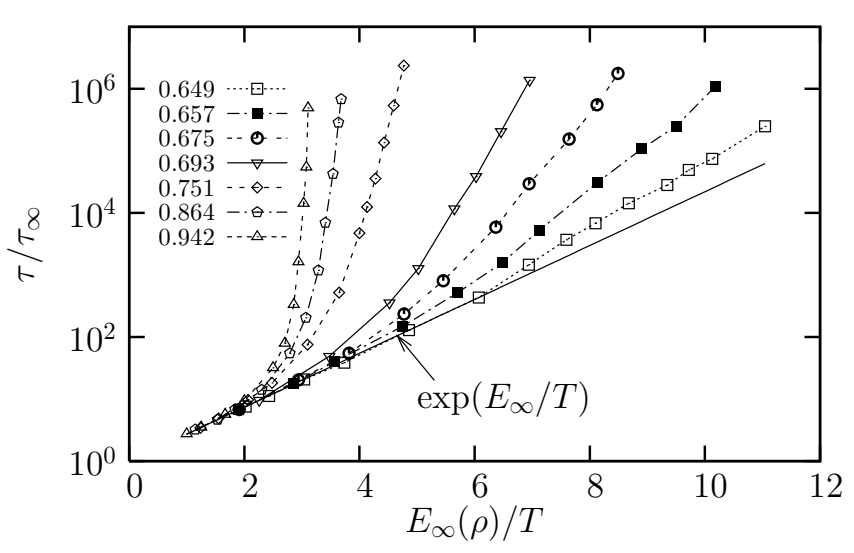

FIG. 11: Rescaling of the relaxation time data for the harmonic repulsive spheres at low temperatures after having normalized the temperature by the energy parameter $E_{\infty}(\rho)$ chosen to make all curves collapse at high- $T$. The density $\rho$ is indicated in the figure. This evidences a dramatic breakdown of density scaling in harmonic spheres, similar to the one observed for the WCA model.

and at odds with the full LJ one, there is no density scaling of the relaxation for truncated harmonic spheres.

The similarity of behavior between the truncated WCA model and the truncated harmonic potential on the one hand and between the LJ model and the repulsive powerlaw model on the other is a strong indication that the presence or absence of density scaling in the dynamics of a glass-former results from the presence or absence of a truncation of all pair interactions at a typical interatomic distance. This observation would perhaps not be very surprising at much lower densities and temperatures (see Fig. 2) since, by construction, the WCA potential coincides with the one of harmonic spheres in Eq. (14) near the cutoff. The surprising feature is that this analogy seems to be relevant up to the liquid densities studied in the present work, and it results in the WCA model behaving differently from the LJ system. As discussed in the following section, this conclusion casts doubts on a description of supercooled liquids in terms of the jamming scenario.

\section{GLASS TRANSITION VERSUS JAMMING PHENOMENON}

The jamming paradigm [19, 44] has been put forward to bring together in a common picture a wide breadth of phenomena and systems involving sluggish dynamics and freezing in an amorphous state. A step to go beyond qualitative comparisons has been taken with the proposal that the slowing down of all jamming systems, whether driven by temperature, density or applied force, is controlled by a zero temperature and zero applied force critical point, "point J" [29]. This proposal has been criticized on several grounds, in particular concerning the uniqueness of point $\mathrm{J}$ itself [45, 46], and the possibility 
that a distinct glass critical point, which could be dubbed "point G", controls the finite temperature dynamics of jamming systems [4]. Here, we focus on the ability of the jamming scenario and of the associated point J (whether unique or not) to describe the slowdown of relaxation of actual glass-forming liquids (and polymers).

A transition near point $\mathrm{J}$ only takes place at zero temperature and moderate density, and it can only be rigorously defined for interaction potentials that are zero beyond some distance and repulsive within it. Therefore, to be applicable to liquids in their (experimentally) accessible range, the jamming scenario must assume that the role of the attractive tail and more generally of longerranged (longer than the typical interatomic distance) interactions is negligible in the physics of the slowing down. To extrapolate the behavior of a real liquid to such conditions, one must then get rid of the attractive forces, which give rise to the gas-liquid coexistence curve and prevent one from taking the liquid to zero temperature below some density (see the phase diagram in Fig. 21), and eliminate the pair interactions beyond some cutoff that physically determines the typical inter-particle distance. Note that this is precisely what the WCA procedure achieves. However, as we have seen above, the behavior of the WCA model strongly deviates, both qualitatively and quantitatively, from that of the LJ model and more generally that of realistic liquids, except at very high densities that are beyond any experimentally accessible range. It is this observation which leads to question the ability of the jamming scenario to describe the dynamics of viscous liquids.

Actually, systems of particles interacting through truncated repulsive interactions, such as the harmonic-like models in Eq. (14) or the WCA model, behave as "effective" hard-sphere systems at low temperature and low pressure. In such thermodynamic conditions, it is the behavior of the pair potential near the cutoff which governs the physics, and if temperature is low, soft repulsive spheres can be seen as "disguised" hard spheres [17, 18. As shown in Fig. 11 and discussed in detail in Ref. [17] this directly implies that the isochoric fragility of such liquids strongly depends on density. This is a first qualitative distinction between the physics near the jamming transition and the one of supercooled liquids.

A second consequence, discussed in Ref. [20], is that relaxation data for soft spheres with truncated repulsive interactions at different state points converge in the low $T$ and low $P$ limit to a hard-sphere-like scaling, such that [20],

$$
\frac{\tau(\rho, T)}{\tau_{\infty}}=\mathcal{H}\left[\frac{P(\rho, T)}{T}\right] .
$$

where $\tau_{\infty}$ has a residual (trivial) dependence on either $T$ or $P($ e.g. in $1 / \sqrt{T}$ or $\sqrt{P})$ that it is now important to take into account as the range of pressure and temperature spanned is very large [compared to the liquid range considered for Eqs. [6 [11)]. The domain of application of this scaling is demonstrated in Fig. 12 where we include

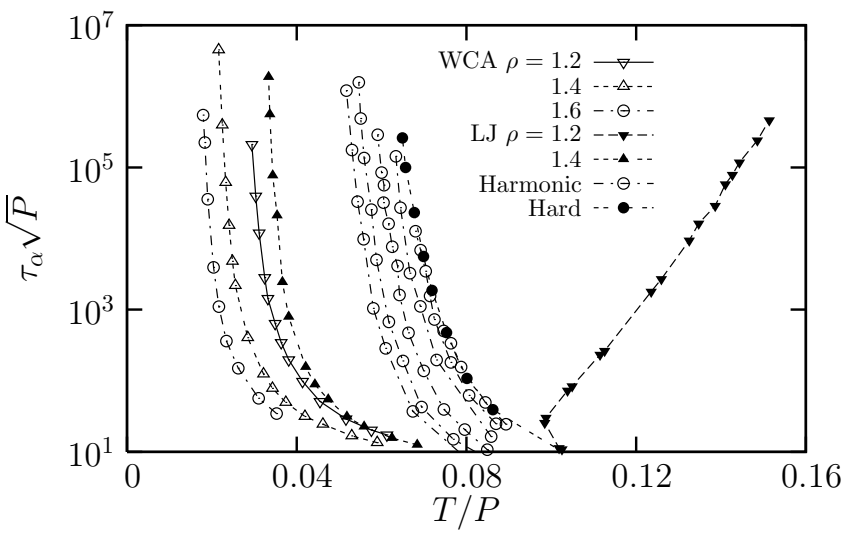

FIG. 12: Hard-sphere-like scaling of the relaxation time. We use Eq. (15) and follow the evolution of the relaxation dynamics from hard spheres [48], to harmonic spheres [17], to WCA and finally LJ systems, which exhibit increasingly larger deviations from hard-sphere behavior.

data obtained from simulations of hard spheres taken from Ref. [48]. The harmonic-sphere data of Fig. 11 indeed converge to the hard-sphere behavior when density is not too large, in agreement with the results of Ref. [20], and display small deviations from Eq. (15) when density is larger. These deviations were empirically addressed in Ref. 17, 18] using a $(\rho, T)$-dependent "effective" volume fraction. Dynamics in such systems with truncated repulsive potentials at low temperature and low-to-moderate pressure seems then dominated by (renormalized) freevolume or congestion effects, as for a hard-sphere fluid. Whether (or not) the slowdown of relaxation is controlled by a $T=0$ point $\mathrm{J}$ [29] or by a $T>0$ point $\mathrm{G}$ [17, 47], the jamming and glass transitions appear in any case strongly intertwined in this case.

However, when moving from harmonic to WCA potentials in Fig. 12, we observe much stronger deviations from the hard-sphere scaling, and the data in fact converge to the vertical axis $T / P=0$ when density increases. This is expected, as in the large density limit we expect the relaxation-time data to be driven by the scaled variable $T^{5 / 4} / P$, which becomes exact for the power law $r^{-12}$ repulsion.

Making the final step from WCA to LJ models in Fig. 12 we realize that the effect of the attractive forces becomes dramatic for normal liquid densities. Since attractive forces induce the presence of the gas-liquid phase separation, the pressure near the coexistence curve drops to zero when temperature decreases much more rapidly than in the absence of the attractive tails. The effect is indeed spectacular for the density $\rho=1.2$ in Fig. 12 since the LJ data have a qualitative behavior opposite to that of truncated purely repulsive systems. This echoes Voigtmann's remarks [28] emphasizing that WCA and LJ models appear more similar when using $(\rho, T)$ than $(P, T)$ variables, for the same reason. This observation is experimentally relevant as atmospheric-pressure data 
for viscous liquids virtually all fall in this regime [28], implying that the hard-sphere-like scaling in Eq. (15) is not obeyed for liquids at normal conditions.

This second argument confirms our claim that in the range of pressure, density, and temperature that corresponds to the actual liquid/supercooled liquid range, the glass transition of the liquid and that of jamming models described by truncated repulsive potentials are different and cannot be controlled by the same $T=0$ point $\mathrm{J}$ critical point.

We finally make the aside that the models defined through truncated soft repulsive potentials differ when considered at high temperature and density. The (unbounded) WCA potential leads to a system that converges to the full LJ model, as shown above; asymptotically, at large $\rho$ and $T$, the WCA and the LJ models behave as a rescaled hard-sphere system (the rescaling is however not the same as at low pressure and temperature). On the contrary, the (bounded) harmonic-like models rapidly reach very high densities at which large numbers of atoms overlap, which seems to confer to these models some sort of mean-field behavior [49], with peculiar physical consequences [50].

\section{CONCLUSION}

Through an extensive comparison of the behavior of a standard Lennard-Jones glass-forming liquid and that of its WCA reduction to a model with truncated pair potentials without attractive tails, we have shown that the slowdown of relaxation is quantitatively and qualitatively different in the two models while the equilibrium pair structure remains very similar. The differences in the dynamics of the two models decrease as density increases but one has to reach unphysically large values of the density for seeing a full convergence of behavior. Clearly, the presence or absence of the attractive tails cannot be neglected in the viscous regime, where a liquid can no longer be simply described according to the conventional van der Waals picture: fluctuations associated with the tails of the interaction potentials do play a role.

At this point, it should be stressed that, in spite of the observed differences, the purely repulsive WCA model is a glass-former, no less than the full LJ one. The attractive tail is not a necessary ingredient to trigger a slowdown of relaxation. The WCA model displays features generically associated with glass formation. For example, as seen from Fig. 5, the associated time dependence of the self intermediate scattering function is characterized by a stretching and the appearance of a plateau as one lowers the temperature. Moreover, the dynamics is increasingly heterogeneous [7] and shows a decoupling between diffusion and structural relaxation, see Fig. 9. Not surprisingly, the temperature dependence of the relaxation time data can also be fitted by the same functional forms, e.g. the Vogel-Fulcher-Tammann formula or a Bässler-type expression [39], for both models. This is enough to invoke analogies, but not necessarily to assign the slowing down to the same mechanisms.

The nonperturbative role of attractive forces may be tentatively attributed to the growth, as temperature decreases, of some form of heterogeneities in the liquid that are not captured by the static pair density correlations. We have not investigated subtler characterizations of the liquid structure, e.g. via higher-order correlation functions or topological measures, but preliminary studies seem to indicate that these are more sensitive to the attractive tails than the pair density correlations and show structural differences between LJ and WCA models that grow as temperature is lowered [40, 51, 52]. This is certainly a worthwhile line of research to pursue.

In trying to characterize the physical significance of the increasing quantitative difference in the dynamics of the full LJ model and of the truncated repulsive WCA one, we have stressed that this difference could not be accounted for by a mere one-parameter rescaling of the data. (The fact that the difference cannot be reproduced either by a large amplification in the dynamics of the small differences in the static pair density correlations is considered elsewhere [27, 34].) The temperature-driven slowdown of relaxation in the two models actually show qualitatively distinct behavior, as manifested by the absence in the WCA model of the density scaling that is found in the full LJ model and in experimentally studied glass-forming liquids and polymers. Moreover, in the LJ and WCA models, this feature appears to be related to the properties of the correlation between the fluctuations of the virial and of the potential energy.

Still concerning the physical significance of our results, we have addressed the question of whether the observed differences between the WCA and the LJ models in the viscous regime are due to the attractive nature of the missing tail (in the WCA model) or to the fact that the tail is truncated at a cutoff corresponding to typical interatomic distances (in the WCA model). We have provided strong evidence that the key feature is the truncation of the interaction potentials and we have stressed that models with truncated repulsive potentials show qualitatively distinct temperature-driven slowing down than glass-forming liquids. This casts serious doubts on the possibility of describing glass formation in liquids by a jamming scenario in which the slowdown of relaxation is controlled by a critical point at zero temperature and density less than liquid densities.

\section{Acknowledgments}

We thank D. Coslovich, D. Reichman and C. P. Royall for fruitful exchanges about this work. L. B.'s work is partially funded by Région Languedoc-Roussillon. 
[1] H. C. Longuet-Higgins and B. Widom, Mol. Phys. 8, 549 (1964).

[2] B. Widom, Science 157, 375 (1967).

[3] J. D. Weeks, D. Chandler, and H. C. Andersen, J. Chem. Phys. 54, 5237 (1971).

[4] J. P. Hansen and I. R. McDonald, Theory of Simple Liquids (Elsevier, Amsterdam, 1986).

[5] D. Chandler, J. D. Weeks, and H. C. Andersen, Science 220, 787 (1983).

[6] J. D. Weeks, Annu. Rev. Phys. Chem. 53, 533 (2002).

[7] D. Chandler, Nature 437, 640 (2005).

[8] J. Kushick and B. J. Berne, J. Chem. Phys. 59, 3732 (1973).

[9] D. P. Dean and J. N. Kushick, J. Chem. Phys. 76, 621 (1982).

[10] L. Berthier and G. Tarjus, Phys. Rev. Lett. 103, 170601 (2009).

[11] C. Alba-Simionesco, D. Kivelson, and G. Tarjus, J. Chem. Phys. 116, 5033 (2002); G. Tarjus, D. Kivelson, S. Mossa, and C. Alba-Simionesco, J. Chem. Phys. 120, 6135 (2004); C. Alba-Simionesco, A. Cailliaux, A. Alegria, and G. Tarjus, Europhys. Lett. 68, 58 (2004).

[12] R. Casalini and C. M. Roland, Phys. Rev. E 69, 062501 (2004); C. M. Roland, S. Hensel-Bielowka, M. Paluch, and R. Casalini, Rep. Prog. Phys. 68, 1405 (2005).

[13] C. Dreyfus, A. Le Grand, J. Gapinski, W. Steffen, and A. Patkowski, Eur. Phys. J. B 42, 309 (2004).

[14] D. Fragiadakis and C. M. Roland, J. Chem. Phys. 134, 044504 (2011).

[15] D. Coslovich and C. M. Roland, J. Phys. Chem. B 112, 1329 (2008)

[16] U. R. Pedersen, T. B. Schroder, and J. C. Dyre, Phys. Rev. Lett. 105, 157801 (2010).

[17] L. Berthier and T. A. Witten, EPL 86, 10001 (2009).

[18] L. Berthier and T. A. Witten, Phys. Rev. E 80, 021502 (2009).

[19] A. J. Liu, S. R. Nagel, W. van Saarloos, and M. Wyart, in Dynamical heterogeneities in glasses, colloids, and granular media Eds.: L. Berthier, G. Biroli, J-P Bouchaud, L. Cipeletti and W. van Saarloos (Oxford University Press, Oxford, 2011).

[20] N. Xu, T. Haxton, A. Liu, and S. Nagel, Phys. Rev. Lett. 103, 245701 (2009).

[21] W. Kob and H. C. Andersen, Phys. Rev. Lett. 73, 1376 (1994).

[22] A. Carré, L. Berthier, J. Horbach, S. Ispas, and W. Kob, J. Chem. Phys. 127, 114512 (2007).

[23] R. Bruning, D. A. St-Onge, S. Patterson, and W. Kob, J. Phys.: Condens. Matter 21, 035117 (2009).

[24] E. A. Mastny and J. J. de Pablo, J. Chem. Phys. 127, 104504 (2007).

[25] V. Testard, L. Berthier, and W. Kob, Phys. Rev. Lett. (in press), arXiv:1101.2293.

[26] S. Sastry, Phys. Rev. Lett. 85, 590 (2000).
[27] L. Berthier and G. Tarjus, Phys. Rev. E 82, 031502 (2010).

[28] T. Voigtmann, Phys. Rev. Lett. 101, 095701 (2008).

[29] C. S. O'Hern, S. A. Langer, A. J. Liu, and S. R. Nagel, Phys. Rev. E 68, 011306 (2003).

[30] S. H. Sung, D. Chandler, and B. J. Alder, J. Chem. Phys. 61, 932 (1974).

[31] N. Lacevic, F. W. Starr, T. B. Schroder, and S. C. Glotzer, J. Chem. Phys. 119, 7372 (2003).

[32] D. Chandler, J. P. Garrahan, R. L. Jack, L. Maibaum, and A. C. Pan, Phys. Rev. E 74, 051501 (2006).

[33] A. Parsaeian and H. E. Castillo, Phys. Rev. Lett. 102, 055704 (2009).

[34] L. Berthier and G. Tarjus, in preparation (2011).

[35] S. Sastry, P.G. Debenedetti, and F. H. Stillinger, Nature (London) 393, 554 (1998).

[36] D. Kivelson, S. A. Kivelson, X.-L. Zhao, Z. Nussinov, and G. Tarjus, Physica A 219, 27 (1995).

[37] D. Chandler and J. P. Garrahan, Annu. Rev. Phys. Chem. 61, 191 (2010).

[38] Y. Brumer and D. R. Reichman, Phys. Rev. E 69, 041202 (2004).

[39] Y.S. Elmatad, D. Chandler, and J.P. Garrahan, J. Phys. Chem. B 114, 17113 (2010).

[40] D. Coslovich, arXiv:1102.5663.

[41] U. R. Pedersen, N. P. Bailey, T. B. Schroder, and J. C. Dyre, Phys. Rev. Lett. 100, 015701 (2008); N. P. Bailey, U. R. Pedersen, N. Gnan, T. B. Schroder, and J. C. Dyre, J. Chem. Phys. 130, 039902 (2009).

[42] N. P. Bailey, U. R. Pedersen, N. Gnan, T. B. Schroder, and J. C. Dyre, J. Chem. Phys. 129, 184508 (2008); T. B. Schroder, N. P. Bailey, U. R. Pedersen, N. Gnan, and J. C. Dyre, J. Chem. Phys. 131, 234503 (2009).

[43] D. Coslovich and C. M. Roland, J. Chem. Phys. 131, 151103 (2009).

[44] A. J. Liu and S. R. Nagel, Nature 396, 21 (1998).

[45] A. Donev, S. Torquato, F. H. Stillinger, and R. Connelly, Phys. Rev. E 70, 043301 (2004).

[46] P. Chaudhuri, L. Berthier, and S. Sastry, Phys. Rev. Lett. 104, 165701 (2010).

[47] G. Parisi and F. Zamponi, Rev. Mod. Phys. 82, 789 (2010).

[48] G. Brambilla, D. El Masri, M. Pierno, G. Petekidis, A. B. Schofield, L. Berthier, and L. Cipelletti, Phys. Rev. Lett. 102, 085703 (2009).

[49] A. Ikeda and K. Miyazaki, Phys. Rev. Lett. 106, 015701 (2011).

[50] L. Berthier, A. J. Moreno, and G. Szamel, Phys. Rev. E 82, 060501(R) (2010).

[51] J. Taffs, A. Malins, S. Williams, and C. P. Royall, J. Chem. Phys. 133, 244901 (2010).

[52] D. Reichman (private communication). 